\title{
Research Advances in Rice Cold Tolerance Gene Mapping
}

\author{
Siqi Zhang, Xiuping Li \\ College of Life Sciences, Jiamusi University, Jiamusi, Heilongjiang, China, 154007
}

Keywords: Rice Cold Tolerance; Gene Mapping; QTL Mapping; Association Analysis; Map Cloning

\begin{abstract}
With the rapid development of molecular biology theory and technical means, rice gene mapping technology is becoming more and more mature. In this paper, the research progress of QTL mapping method, rice cold tolerance correlation analysis, rice cold tolerance gene fine mapping and map cloning were reviewed in order to provide reference for future research on cold tolerance of rice.
\end{abstract}

\section{Introduction}

Rice chilling injury refers to the impact of low temperature caused by rice cannot be healthy growth and development of the phenomenon, which seriously affected China and the world rice quality and yield, food safety and stability of a certain threat. The resistance of rice to chilling injury is called cold tolerance, and the effective measures to help cultivate cold tolerant cultivars by molecular biology are rice cold tolerance genes.

\section{Identification and Evaluation of Rice Cold Tolerance}

The results showed that the rice varieties with different cold tolerance and the cold tolerance were selected for the purpose of chilling injury identification. In 2003, Andaya V C et al. [1] summarized the method of identification of cold tolerance of rice, and then Han Longzhi [2] also summarized the cold tolerance identification method, cold tolerance index and grading standard of rice used at home and abroad. Zhang Jingyong [3] the rice cold method is divided into direct identification and indirect identification method. In the rice cold tolerance identification facilities, Li Dalin [4] concluded that in the commonly used cold tolerance identification facilities, artificial climate chamber and artificial climate chamber is more suitable for the need for accurate data experiments, rough identification and large sample Screening is more suitable for cold water irrigation.

Identification of Cold Tolerance at Different Growth Stages. The cold tolerance identification method and the cold tolerance identification index of rice were different in different growth and development periods. For example, $\mathrm{Fu}$ Tailu et al. [5] identified seedling cold tolerance identification of 21 rice plants with seedling survival rate as identification index. The results showed that at low temperature there was no significant correlation between seedling height and root survival rate, but it was unreasonable to use the survival rate as the identification index of rice seedling.

In the early stage of rice fertility, the tolerance characters were mainly divided into cold tolerance and cold tolerance at bud stage. Chen et al. [6] called generalized cold tolerance, including bud stage and germination stage. According to Ogawa $\mathrm{M}$ et al. [8], the cold tolerance of 10 cold tolerant rice cultivars was identified. The results showed that different temperatures had significant correlation to germination rate. Through this experiment, The optimum temperature for the identification is $14-20^{\circ} \mathrm{C}$. Identification of cold tolerance of rice germination period can refer to the method of Li Taigui et al. [9]. The method of identification of cold tolerance in bud stage is usually based on the literature of Han Longzhi [10], Cunying Mountain [11] and Qiao Yongli [12].

The early identification index of rice bud stage is its low seedling rate. XIA Xiu-zhong et al. [13] 
identified 419 rice cultivars in Guangxi with budding rate and cold tolerance at seedling stage. The results showed that the cold tolerance of bud stage was weak, and the bud stage And seedling stage showed strong cold resistance.

Appropriate improvement in the identification method can reduce the error on the budding rate, such as Xiao Yulong et al. [14] refine the criteria for the cold tolerance of rice bud stage used by Liu [15], calculate the cold tolerance of 23 test materials, the results show that this improved evaluation method reduces the experimental error.

Leaf redness is often used as an indicator of cold tolerance in rice seedling. Wang Shangming et al. [16] identified Ganzairea indica 49 and Dongye 1 and Japonica 0298 / Dong Ye 1, and then carried out the identification of cold tolerance at seedling stage of hybrid progeny. The results showed that the cold tolerance No. 1 of the cold resistance stronger. Song Jiaoyun et al. [17] identified and evaluated the cold tolerance of 150 core rice germplasm populations at seedling stage. The results showed that the cold tolerance at seedling stage showed a normal distribution trend. He Ping [18] identified and evaluated the cold tolerance of 3-4 leaf stage seedlings, the results showed that the cytoplasm and nuclear genes have affected the cold tolerance of rice sterile lines. Zhou Yong et al [19] to low temperature treatment of rice seedling survival rate as an identification index, the use of chromosome fragment substitution system identified a seedling cold resistance QTLqCTS3-1. Zou De-tang et al. [20] identified the cold tolerance of Dongnong 422 and Wuzhan 131 at the tillering stage. The results showed that the cold tolerance of Zengyu 131 was stronger than that of Dongnong 422. Hu Xiaochen [21] and other 95 kinds of rice germplasm resources were identified at the seedling stage cold tolerance, 10 seedling cold and 9 cold sensitive materials were screened out. PENG Qiang et al. [22] identified the cold resistance of 213 RILs with seedling rate as the identification index, and the results showed that the parent Asominori was a strong cold resistant variety.

Rice booting at the flowering period is often used as a cold tolerance identification index. The method of identification of cold tolerance is mainly for 7 days, natural low temperature sowing method, circulating cold water irrigation method and so on. Zhang Sanyuan et al. [23] had identified the cold tolerance of brittle rice varieties in Jilin rice varieties, and found that 397, Kyrgyzstan 94-B2 and Changbai 7 were characterized by strong cold tolerance. Wang Jinming [24] identified 230 rice germplasm resources at the booting stage, and screened 43 tobacco resistant germplasm resources. Cui Di [25] evaluated the cold tolerance of 347 rice materials in 11 provinces and 9 other countries, and proved that the cold tolerance of rice at booting stage in Heilongjiang area had a good genetic basis.

\section{QTL Positioning for Rice Cold Tolerance}

Quantitative trait (QTL) mapping is a mapping method. The development of DNA marker technology has made great contributions to the genetic research of quantitative traits and its labeling method has also gone through a development stage.

Molecular Markers Which Gene Mapping Often Used. Due to the complexity of the traditional genetic research methods and the errors caused by external factors, the molecular marker technology has become the most effective molecular marker method, which has been obtained in genetic map construction, gene mapping and molecular marker assisted breeding..

The genetic map of the crop is based on RFLP (restriction fragment length), SSR (simple sequence repeat, simple sequence repeat, also known as microsatellite DNA), SPAR (single primer amplification reaction, single primer Amplification reaction) and other markers. RFLP markers are the earliest applied DNA molecular marker technology. SSR markers can detect a single multi-allele locus compared to other markers, commonly used in rice gene mapping. Chen et al. [26] found SSR markers RM280 and RM377 linked to cold tolerance by locating cold tolerance genes on chromosomes. Qiao Yongli [27] QTL analysis of cold tolerance at bud stage was carried out using SSR markers. Three QTLs for cold tolerance were detected. The use of traditional genetic markers for QTL mapping has a large workload, long cycle and other issues, and the third generation of molecular marker SNP (single nucleotide polymorphism) marker development to solve these 
problems, and other repeat sequences the genetic stability of SNP markers is higher than that of morphological markers. The results showed that the SNPs detected by the SLAF marker were evenly distributed on the chromosome. The results showed that the SLAF-seq library was used to construct the SLAF-seq library. Huxiao Chen [29] successfully identified 10 SNP loci between the cold resistant varieties Lijiang New Group Black Valley (LTH) and the cold resistant material IR29, and designed OsSADMC functional marker SADMC-CAPS1 according to one of the SNP loci, the marker could accurately identify the genotype and cold tolerance of OsSADMC with different cold tolerance rice germplasm resources. Charles A. Jos et al. Used the 717 stable high-generation lines from the same indica-japonica combination and 265 uniformly distributed SNP markers to detect the genetic overlap QTLs affecting the stable expression of biological and abiotic resistance. As a result, 18 were detected resistant strains [30].

Mapping of QTLs at Different Growth Stages. Rice cold tolerance is a complex trait controlled by multiple genes. Rice cold tolerance at the same developmental stage is controlled by multiple cold tolerance QTLs, and the environment also affects the expression of cold tolerance. A number of QTLs related to cold tolerance have been identified. Lin et al. [31] used Nipponbare as the donor parent, 9311 as the recipient parent, and constructed 1500 BC4F1 populations. Four QTLs for cold tolerance were detected. Through comparison and analysis, eight cold-resistant QTLs were detected. Yang YFeng [33] The 192 cold tolerance QTLs were integrated on the same map, and the chromosomal location relationships of cold tolerance QTLs at different developmental stages and during the same developmental period were compared. The results showed that the cold tolerance of rice was controlled by different QTLs at different times; Qqin et al. [34] used four QTLs related to cold tolerance at seedling stage on four chromosomes of indica-japonica crossbred DH population. Yang Luomiao et al. [35] constructed 190 juvenile inbred lines with Donggan 422 and Longqing 131, and set up control and cold water stress for three consecutive years. The effects of different environments on the traits were evaluated by GGEbipiolt and the correlation between traits. Andyal [36] has mapped the QTLs for cold tolerance at the booting stage of rice. The cold tolerance QTLs are almost distributed in the whole genome, indicating that the cold tolerance at booting stage is a very complex quantitative trait. (NGS-BSA), which solves the problem that the traditional QTL method is often time-consuming and the result is not accurate and so on. In addition, the two-generation sequencing technology (NGS) combined with the separation and separation method (NGS-BSA) Locate the QTL. Liu Tao et al [38] collected 11 mapping groups, summed up a total of 96 rice seedling cold tolerance QTL, and Biomercator2.1 and common marker mapping QTL integrated into the reference map corne112001, through the meta-analysis method the QTLs of QTLs were established, and 18 "real QTLs" were identified and their linkage markers were established.

\section{Correlation Analysis of Rice Cold Tolerance}

The association analysis, also known as linkage disequilibrium mapping, can be directly identified by molecular markers and target trait polymorphisms, which directly identify genes that are significantly related to the ideal trait. (Q) and MLM (Q + K) were used to analyze the cold tolerance of 150 rice plants. The results showed that 84 marker sites were detected by the method of GLM (Q) and MLM (Q + K) in TASSEL2.1R software. The results showed that there were five correlations with the cold tolerance of the bud stage, and the relationship between the cold tolerance and the cold tolerance at the seedling stage was analyzed by the same method. Cold resistance associated with the site. Yang et al [41] using TASSEL2.1 software GLM method to mark and seedling cold tolerance correlation analysis, obtained 10 and bud stage and seedling cold tolerance genes are associated with markers. Zhang Yanmei et al. [42] identified 140 cold-tolerant differences in cold tolerance by using 84 SSR markers for 140 samples of northeast japonica rice cultivars as a material. The SSR markers were associated with a total of 25 significant correlations the largest rate is $29.24 \%$, the results show that RM214 a total of 8 kinds of markers can be used as a resource selection. 


\section{Fine Mapping and Map Cloning of Rice Cold Tolerance Gene}

Rice cold tolerance is the quantitative trait of polygene control. Because of the difference of environment and the number of gene population, the accuracy of localization is not very high. It is not easy to determine whether a major QTL is a major gene or the effect of a micro-gene interaction. Therefore, it is necessary to carry out the fine mapping of genes, and on the basis of fine positioning on the cold tolerance of rice for map cloning. Li et al. [43] constructed a set of indica and japonica recombinant inbred lines USSR5 and N22 mapping groups. Under the low temperature condition, the germination ability of rice was identified, and three low-temperature germination QTLs were obtained. Xiao Ning et al. [44] constructed the BC2F1 locus using Dongxiang wild rice as parent and Nanjing 11, and located seven QTLs related to cold tolerance on four different chromosomes. The QPSR2-1, qLOP2 and qRC10-2 loci were fine mapped and the Os02g0677300 could be the best candidate for qPSR2-1 and qLOP2. The results showed that the QPSR2-1, qLOP2 and qRC10-2 loci were well located in the QTLs with the contribution rate of more than $10 \%$ gene. Mapping cloning is the most effective way to clone quantitative trait genes. Saito et al. [45] mapped a cold-resistant QTL Ctb1 to a physical distance of $17 \mathrm{~kb}$ and cloned it. The results showed that the ubiquitin-proteasome pathway may be involved in the cold-resistant process at booting stage. Wang Lan [46] using the cold-resistant varieties of Japanese sunny and a cold-resistant variety V216 as a test group, through the map cloning technology to obtain the gene, and named CORI. Li Guangxian et al. [47] performed chromosomal fine loci on QTLqHD3 at heading stage of rice, and finally found 7 expressible gene clones in about $62.4 \mathrm{~kb}$ chromosome interval between RM14314 and RM14320. Chen Junyu et al [48] developed a new QTL fine positioning strategy, which is based on SeqRHs (ladder residual hybrids) positioning method, has been through the rice resistance to cold tolerance gene complementary test proved.

\section{Conclusion}

Through the research on the progress of assisted breeding by molecular biology techniques, it has been found that the researchers have been exploring the cold tolerance problem of rice, and the gene localization technology has been widely used in the breeding of plant resistance. There are many studies on QTLs for cold tolerance in rice at home and abroad, but there are still few studies on fine loci and map cloning. Due to the complexity of cold tolerance genes, the fine positioning of cold tolerance genes is difficult, and it is necessary to explore more and more efficient rice cold tolerance gene mapping strategy, in order to achieve the purpose of actual breeding.

\section{References}

[1] Andaya V C, Mackill D J.QTLs conferring cold tolerance at the booting stage of rice using recombinant inbred lines from a japonica $\mathrm{x}$ indica cross. Theor Appl Genet, 2003, 106(6):1084-1090

[2] Han Longzhi, Zhang Sanyuan. Evaluation method of cold tolerance of rice. 2004, 5 (1): 75-80

[3] Zhang Jingyong.Study on Cold Tolerance Identification Methods of Rice[J]. Yanbian University, 2006

[4] Li Dalin, Yang Qing, Ma Wendong, etc. Rice cold tolerance identification facilities and application[J]. Northern rice, 2015, (06): 44-46

[5] Fu Tailu. Rice cold tolerance at different growth stages and identification of identification indicators[D]. Sichuan Agricultural University, 2008

[6] Chen Jing. Identification of Cold Tolerance of Rice Germplasm and Cloning of OsNADPH1 in Rice Stress Response[D]. Sichuan Agricultural University, 2006

[7] Zhang Jingyong. Rice cold resistance identification method[D]. Yanbian University, 2006 
[8] Ogawa M,Hanada A,Yamaguchi Y.Gibberellin biosynthesis and response during Arabidopsis seed germination.Plant Cell.2003.15:1591-1640

[9] Li Taigui, an indoor method for screening cold-tolerant varieties of rice at different growth stages at low temperature [J]. Foreign Agricultural Science and Technology, 1984 (4): 18-21

[10] Han Longzhi, Zhang Sanyuan. Evaluation method of cold tolerance of rice. 2004, 5 (1): 75-80

[11]Ying Cunshan. China's rice resources research progress [J]. Chinese Journal of Rice Science, 1992, (03): 142-144.

[12] Qiao Yongli, Han Longzhi, An Yungping et al. Molecular Mapping of QTLs for Cold Tolerance at Bud Stage [J]. Chines Agricultural Sciences, 2005, (02): 217-221.

[13] Xia Xiu-zhong, Zhang Zong-qiong, Yang Xing-hai et al. Identification and Correlation Analysis of Cold Tolerance of Rice Varieties in Guangxi[J]. Acta Metallurgica Sinica, 2016, (06): 969-975.

[14] Xiao Yulong, Wang Zhiquan, Lei Jianguo, etc. A method for the accurate identification and evaluation of cold tolerance in rice bud stage [J]. Journal of Jiangxi Agricultural Sciences, 2014, (11): $1-5$.

[15] Liu H L,Sun S C,Wang J G,et al. Identification of Cold Tolerance of Rice Germplasm Resource at Germplasm Resource at Germinating Stage in Cold REGION [J] .Journal of Northeast Agricultural University,2008,15(2):1-4.

[16] Wang Shangming, He Haohua, Xiao Yeqing. Genetic Analysis of Chilling Tolerance at Seedling Stage in Dongye No.1, Rice [J] .Hubei Agricultural Sciences, 2008, (01): 1-4.

[17] Song Jianyu, Zhao Minghui, Li Jinquan, etc. Using association analysis to excavate rice cold tolerance genes and cold tolerance germplasm materials [A]. China Crop Society. 2014 China Crop Society Academic Conference Proceedings[C]. China Crop Society: 2014: 1.

[18] He Ping, Chen Xionghui, Duan Zhongai, Xu Yong, Fu Xuelin.Calculation of Cold Tolerance and Physiological and Biochemical Characteristics of High Quality Wheat Lines in Seedling Stage [J]. Chinese Agricultural Science Bulletin, 2012, (36): 34-40.

[19] Zhou Yong, Wang Zhongde, Tao Yajun, etc. Identification and molecular mapping of QTLqCTS3-1 related to cold tolerance in rice seedling. Journal of Jiangsu Agriculture, 2013,29 (6): 1181-1186

[20] Study on QTL Mapping of Cold Tolerance at Tillering Stage in Rice under Cold Water Stress. Journal of Northeast Agricultural University, 2012.43 (10): 96-102

[21] Hu Xiaochen, Zhang Ting, Yang Sheng et al. Development and utilization of OsSADMC functional markers for rice cold stress response gene [J]. China Journal of Rice Science, 2015,29 (5): 475-480

[22] Peng Qiang, Zhang Da-shuang, Wu Jian-qiang, et al.Characteristics Analysis of QTLs for Chilling Tolerance at Seedling Stage in Rice [J]. Guizhou Agricultural Sciences, 2015, (05): 11-13 + 18

[23] Zhang Sanyuan, Zhang Junguo, Zhao Jinsong, etc. High quality, high yield, multi-resistant rice varieties Jijing 88 breeding research [J]. Crop Research, 2007, (03): 222-226.

[24] WANG Jin-ming, YANG Chun-gang, JIN Guo-guang, SUN Qiang, LIN Xiu-yun. Identification of Cold Tolerance at Germination and Booting Stage of Rice Germplasm Resources [J]. Northeast Agricultural Sciences, 2016, (02): 1-4.

[25] Cui Di, Yang Chungang, Tang Cuifeng, et al.Evaluation of cold tolerance traits of japonica rice cultivars under low temperature stress [J]. Acta Metallurgica Sinica, 2012, 13 (5): 739-747 
[26] CHEN Da-zhou, ZHONG Ping-an, XIAO Ye-qing et al. Using SSR markers to locate cold tolerance genes in Dongxiang wild rice [J] .Chinese Journal of Rice Science, 2002, 24 (6): 735-756

[27] Qiao Yongli, Molecular characterization of QTLs for cold tolerance in rice buds. 2005, 38 (2): 217-221

[28] Song Jianyu, Zhao Minghui, Li Jinquan, etc. Using association analysis to excavate rice cold tolerance genes and cold resistant germplasm materials [A]. Chinese Crop Society. 2014 China Crop Society Academic Conference Proceedings [C]. China Crop Society : 2014: 1.

[29] Hu Xiaochen, Zhang Ting, Yang Sheng et al. Development and utilization of OsSADMC functional markers for rice cold stress response gene .2015,29 (5): 475-480

[30] Wang Z.F.,Wang F.H.,Zhou R.,2011,Identification of quantitative trait loci for cold tolerance during the germination and seeding stages in rice(Oryza sative L.),Euphytica,181(3):405-413

[31] Lin J,Zhu W Y,Zhang Y D,et al.Detection of QTL for cold tolerance at budbursting stage using chromosome.Segment substitution lines in rice(Oryza sativa L.).Rice Sci, 2011, 18:71-74

[32] Zhu Jinyan, Yang Mei, etc., using the chromosome single-segment substitution line to locate the rice buds cold tolerance QTL.2015,50 (3): 338-345

[33] Yang Tai-feng, Zhang Shao-hong, Zhao Jun-liang, Huang Zhang-hui, Zhang gui-yuan, Liu Bin.Comparative analysis of QTLs for cold tolerance in rice [J]. Molecular Plant Breeding, 2015, (01): 1-15.

[34] Qian Qian, had vigorously, He Ping and so on. QTLs for Chilling Tolerance of Maize Indica and Japonica Crosses at Seedling Stage[J]. Chinese Science Bulletin, 1999,44 (22): 2402-2408

[35] Yang Luomiao, Sun Jian, Zhao Hongwei, Wang Jingguo, Liu Huilong, Zou Detang.QTL Analysis of Heading and Yield Traits in Rice under Cold Water Stress in Different Years [J]. Chinese Journal of Agricultural Sciences, 2016 (18): 3489-3503.

[36] Andayal VC,Tai TH.Fine mapping of the q CTS12locus,a major QTL for seeding coldtolerance in rice.Theoretical and Applied Genetics113.2006

[37] YANG Ze-mao.Characteristics of QTLs for Cold Tolerance and SMS in Male Sterile Genes [J]. Journal of Zhejiang University, 2013

[38] LIU Tao, WANG Jing-guo, LIU Hua-long, ZOU De-tang.Study on Cold Map QTL Mapping at Seedling Stage [J]. Acta Agronomica Sinica, 2010, (03): 15-19.

[39] Song Jianyu, Zhao Minghui, Li Jinquan, etc. Using association analysis to excavate rice cold tolerance genes and cold resistant germplasm materials [A]. China Crop Society. 2014 China Crop Society Academic Annual Conference Proceedings C]. China Crop Society: 2014: 1.

[40] Sun Guiyu. Cold-type Japonica Rice Early Cold Tolerance Identification and SSR Marker Linkage Analysis[D]. Northeast Agricultural University, 2015

[41] YANG Yan-hua.Using association analysis to find out the allelic variation of cold tolerance in early rice germination [D]. Heilongjiang University, 2013

[42] Zhang Yanmei, Zou Detang. Identification of Cold Tolerance and SSR Marker Correlation Analysis of Northeast Japonica Rice at Tillering Stage[J]. China Journal of Rice Science, 2012, (04): 423-430.

[43] Li LF,Liu X,Xie K,qLTG-9,a stable quantitative trait locus for lowtemperature germination in rice (Oryza sativa L.).Theor Appl Genet.2013 126, 2313-2322

[44] Xiao Ning. Dongxiang wild rice seedling cold resistance QTLs fine positioning and cloning [D]. Yangzhou University, 2015

[45] Saito K,Hayano-Saito Y,Kuroki M,Sato Y.Map-based cloning of the rice cold rolerance gene 
Ctb1.Plant Sci 2010,97-102

[46] WANG Lan, HUANG Li-chao, DAI Li-ping et al. Mapping QTLs for Leaf-shaped Traits at Maturity Stage in Rice (Oryza sativa L.) Using Japanese Cabbage / 9311 Recombinant Inbred Lines [J]. Chinese Journal of Rice Science, 2014, (06): 589-597

[47] Li Guangxian, Yao Fangyin, Hou Hengjun. Fine Mapping of QTLqHD3 in Heading Stage of Rice. 2015, 29 (10): 1845-1851

[48] Chen Junyu, Guo Liang, Wang Linlin, et al.Characteristics of QTLs for QTLs Based on Ladder Dependent Hybrid [A]. Chinese Journal of Genetics. The sixth National Symposium on Quantitative Genetics of Animals and Plants [C]. Genetics Society: 2014: 1 